\title{
Green's function for multielectron ions and its application to radiative recombination involving dielectronic recombinations
}

\author{
X. M. Tong, ${ }^{1,2, *}$ N. Nakamura, ${ }^{3}$ S. Ohtani, ${ }^{3,4}$ T. Watanabe, ${ }^{3,5}$ and N. Toshima ${ }^{1}$ \\ ${ }^{1}$ Institute of Materials Science, Graduate School of Pure and Applied Sciences, University of Tsukuba, 1-1-1 Tennodai, Tsukuba, Ibaraki \\ 305-8573, Japan \\ ${ }^{2}$ Center for Computational Sciences, University of Tsukuba, 1-1-1 Tennodai, Tsukuba, Ibaraki 305-8577, Japan \\ ${ }^{3}$ Institute for Laser Science, The University of Electro-Communications, Chofu, Tokyo 182-8585, Japan \\ ${ }^{4}$ CREST, Japan Science and Technology Agency, Chofu, Tokyo 182-8585, Japan \\ ${ }^{5}$ Department of Physics, Tokyo Metropolitan University, 1-1 Minami-Osawa, Hachioji, Tokyo 192-0364, Japan
}

(Received 23 March 2009; published 6 October 2009)

\begin{abstract}
We propose a general method to calculate the full Green's function of multielectron atomic ions. The key point exists in the usage of $L^{2}$ integrable functions as a complete basis set in a finite region together with an optical potential to guaranty the outgoing scattering boundary condition. In such a way, the cumbersome procedure of adjusting boundary conditions in solving the differential Schrödinger equation is avoided. To show the validity of the method, we studied the radiative recombination involving dielectronic recombinations of Be-like $\mathrm{Hg}(\mathrm{Z}=80)$ ions. The radiative damping effect is taken into account naturally in the present method. The calculated results reproduce well the asymmetric line profile observed in the experiments.
\end{abstract}

DOI: 10.1103/PhysRevA.80.042502

\section{INTRODUCTION}

Green's function plays an important role in understanding many dynamic processes in atomic systems. Direct calculation of the Green's function is a powerful and rigorous theoretical way but its application has been so far limited to single electron or quasi-single-electron systems $[1,2]$ because huge computational resources are required for multielectron systems. In principle, all the scattering processes can be studied by solving the following Schrödinger equation

$$
H \Psi^{+}=E \Psi^{+},
$$

under proper scattering boundary conditions. Here, $H$ is the Hamiltonian of the total system, $E$ the collision energy, and $\Psi^{+}$the wave function satisfying a given scattering boundary condition. The above equation can be solved by the R-matrix method [3], for instance, if not many open channels are involved. Note that the R-matrix has a numerical difficulty when the radiative damping is important [4]. Solving directly the above equation for multielectron ions is still a challenging work when many open channels are coupled.

Alternatively, the scattering wave function can be written in an integral form as

$$
\left|\Psi^{+}\right\rangle=\left|\Psi_{0}\right\rangle+\frac{1}{E-H+i \eta} V\left|\Psi_{0}\right\rangle=\left|\Psi_{0}\right\rangle+G^{+}(E) V\left|\Psi_{0}\right\rangle .
$$

Here, $\Psi_{0}$ is the initial state for a given scattering process, $V$ the residual interaction absent in the initial channel, and $\eta$ a positive infinitesimal to ensure the outgoing boundary condition. Different from the differential Eq. (1), the boundary conditions are already taken into account fully in the integral Eq. (2). The radiative decay can be included by making the energy complex if necessary. Equation (2) can be further transformed into a time-dependent equation, which was

\footnotetext{
*tong@ims.tsukuba.ac.jp
}

PACS number(s): 32.70. -n, 31.15.-p, 25.40.Lw, 32.80.Zb

solved to study the protonium formation for antiproton collisions with hydrogen atoms [5]. The key ingredients of the time-dependent approach are (1) to discretize the space on the pseudospectral grid for forming a complete basis set $\left(L^{2}\right.$ integrable basis), (2) to use an energy dependent optical potential for filtering out the outgoing flux and guarantying the outgoing boundary conditions, and (3) to calculate the scattering wave function by a time-propagation method.

We can use the same approach [6,7] basically to investigate other dynamic processes. But for some processes, for example, the radiative recombination (RR) correlated with the dielectronic recombination (DR), it is not practical to carry out the time-propagation directly because of the two reasons. (1) Highly accurate calculations are required because interference effects induce delicate cancellation and (2) calculations have to be done at many energy points in order to resolve the energy spectra at fine intervals. These make the computational time extremely long. In this paper, we propose an alternative method to solve Eq. (2) without the time propagation, yet keeping the first two key ingredients. We present our theoretical method in Sec. II and an application of the method to the radiative combination in Be-like $\mathrm{Hg}$ ions in Sec. III.

\section{THEORETICAL METHOD}

The relativistic Hamiltonian of the $N$-electron system may be written as (atomic units $m=\hbar=e=1$ are used throughout unless otherwise stated)

$$
\begin{aligned}
H= & \sum_{i=1}^{N}\left(c \boldsymbol{\alpha}_{i} \cdot \mathbf{p}_{i}+\beta_{i} c^{2}-\frac{Z}{r_{i}}\right)+\sum_{i<j} V\left(\mathbf{r}_{i}, \mathbf{r}_{j}\right)=\sum_{i=1}^{N} h_{i} \\
& -\sum_{i=1}^{N}\left(V_{e f f}\left(r_{i}\right)+\frac{Z}{r_{i}}\right)+\sum_{i<j} V\left(\mathbf{r}_{i}, \mathbf{r}_{j}\right)=\sum_{i=1}^{N} h_{i}-V_{r e s}(N) .
\end{aligned}
$$

Here, $c$ is the velocity of light, $\boldsymbol{\alpha}, \beta$ the Dirac matrices, $Z$ the atomic number, $V_{\text {res }}(N)$ the residual interaction and $i, j$ are 
the electron indices. $h_{i}$ is a single-electron Dirac Hamiltonian defined as $h_{i}=c \boldsymbol{\alpha}_{i} \cdot \mathbf{p}_{i}+\beta_{i} c^{2}+V_{\text {eff }}\left(r_{i}\right)$, with $V_{e f f}(r)$ the effective potential, which was obtained from the relativistic density functional theory with the self-interaction correction [8]. $\boldsymbol{\alpha}_{i}$, and $\beta_{i}$ operate on the spinor components of the $i$-th electron. $V\left(\mathbf{r}_{i}, \mathbf{r}_{j}\right)$ stands for the electron-electron interactions which include both the Coulomb and the generalized Breit interactions (GBI) [9]. The expression of $V\left(\mathbf{r}_{i}, \mathbf{r}_{j}\right)$ used in the present work is

$$
\begin{aligned}
V\left(\mathbf{r}_{i}, \mathbf{r}_{j}\right)= & \frac{1}{r_{i j}}-\boldsymbol{\alpha}_{i} \cdot \boldsymbol{\alpha}_{j} \frac{\cos \left(\nu r_{i j}\right)}{r_{i j}}+\left(\boldsymbol{\alpha}_{i} \cdot \nabla_{i}\right) \\
& \times\left(\boldsymbol{\alpha}_{j} \cdot \nabla_{j}\right) \frac{\cos \left(\nu r_{i j}\right)-1}{\nu^{2} r_{i j}},
\end{aligned}
$$

where $\mathbf{r}_{i j}=\mathbf{r}_{i}-\mathbf{r}_{j}$ and $\nu$ is the virtual photon energy divided by $c$. If we choose $\nu=0$, we get the Breit interaction (BI0) in the low-frequency limit.

To calculate the Green's function of the $N$-electron system, we first prepare single-electron wave functions $\left\{\phi_{i}\right\}$ by solving the following Dirac equations

$$
h \phi_{i}=\left[c \boldsymbol{\alpha} \cdot \mathbf{p}+\beta c^{2}+V_{e f f}(r)\right] \phi_{i}=\epsilon_{i} \phi_{i},
$$

on a generalized pseudospectral grid $[10,11]$ within a sphere of a radius $r_{\max }$. The wave functions $\left\{\phi_{i}\right\}$ form a complete basis set within the sphere. We divide the wave functions into two groups, bound states with $\epsilon_{i}<c^{2}$ and continuum states with $\epsilon_{i}>c^{2}$. Note that the eigenvalue $\epsilon_{i}$ contains the rest energy $c^{2}$. The positron solutions of the above equation are not used in the subsequent calculations. From the singleelectron wave functions $\left\{\phi_{i}\right\}$, we construct a configuration wave function of the $N$-electron system. The configurations are divided into two types, bound-type configurations $\left\{\Phi_{b, i}\right\}$ where all the electrons are in bound states and continuumtype configurations $\left\{\Phi_{c, j}\right\}$ where one electron is in a continuum state and all others are in bound states. The configurations with the same $(N-1)$-electron core structure and a different continuum wave function with the same $\ell, j$ form an ionization channel. Here, $\ell, j$ are the single-electron orbital and total angular momenta. After adding an optical potential $V_{o p t}$ to $V_{e f f}$, the matrix elements of the single-electron operator $h$ are written as

$$
\begin{gathered}
\left\langle\phi_{i}|h| \phi_{j}\right\rangle=\tilde{\epsilon}_{i} \delta_{i j}, \\
\text { with } \tilde{\epsilon}_{i}= \begin{cases}\epsilon_{i} & \text { for bound states } \\
\epsilon_{i}-i \gamma_{i} & \text { for continuum states }\end{cases}
\end{gathered}
$$

and $\gamma_{i}=\left\langle\phi_{i}\left|V_{o p t}\right| \phi_{i}\right\rangle$. The energy dependent optical potential $V_{\text {opt }}$ is expressed as

$$
V_{o p t}(\epsilon, r)=\left\{\begin{array}{cc}
0 & \text { for } r<r_{c} \\
V_{0}\left(\frac{r-r_{c}}{r_{\max }-r_{c}}\right) & \text { for } r \geq r_{c}
\end{array}\right.
$$

with $\epsilon^{1 / 2} /\left(r_{\max }-r_{c}\right)<V_{0}<\epsilon^{3 / 2}\left(r_{\max }-r_{c}\right)[5,12]$. Here, $r_{c}$ is the starting position of the optical potential. Mathematically, introducing $\gamma_{i}$ is equivalent to adding a filter to absorb the outgoing wave [7]. Using a unified notation $\left\{\Phi_{k}\right\}$ to represent both the bound configurations $\left\{\Phi_{b, i}\right\}$ and the continuum con- figurations $\left\{\Phi_{c, j}\right\}$, we get the matrix elements for configuration mixing as

$$
H_{k, k^{\prime}}=\left\langle\Phi_{k}|H| \Phi_{k^{\prime}}\right\rangle .
$$

The coupling coefficients of the spin-angular momenta and the angular coefficients of the multipole transition matrix elements are calculated by ANCO package by Gaigalas and Fritzsche [13]. Note that the $H$ matrix is not Hermitian but symmetric complex since we have introduced the complex optical potential $V_{\text {opt }}$ and it contains both diagonal and offdiagonal matrix elements of the Breit interaction. Diagonalizing the $H$ matrix, we obtain the eigenenergies $\left\{E_{i}\right\}$ and the eigenfunctions $\left\{\Psi_{i}=\Sigma_{k} A_{i k} \Phi_{k}\right\}$, which are calculated once for all. The Green's function of the scattering system with total scattering energy $E$ is expressed as

$$
G^{+}(E)=\sum_{i}\left|\Psi_{i}\right\rangle \frac{1}{E-E_{i}+i \eta}\left\langle\Psi_{i}\right| .
$$

The dynamic processes can be studied by the Green's function once we obtain $\left\{\Psi_{i}\right\}$ and $\left\{E_{i}\right\}$.

To test the idea, we calculated the photoionization cross sections of hydrogen atoms by the present Green's function method. The calculated photoionization cross sections are in agreement with the analytical ones within $1 \%$ when the photoelectron energies are of the order of the ionization potential and the agreement becomes better for lower photoelectron energies. In the present study of the radiative recombination, the electron energy is comparable to or smaller than the ionization potential and thus we can expect that Eq. (9) gives accurate reliable results. The idea of the combination of an $L^{2}$ basis set and an optical potential to study the resonant process might be related to the complex scaling method [14-16] in some respects but the present method is more general and it can be easily extended to multielectron systems.

\section{RESULTS AND DISCUSSION}

Once we obtain the full Green's function, we can extract information of various dynamics. Taking Be-like $\mathrm{Hg}$ ions as an example, we study the RR process involving DR resonances via $1 s 2 s^{2} 2 p_{1 / 2} 2 p_{3 / 2}$ configurations, which was investigated experimentally [17]. The configurations included in the calculations are $1 s^{2} 2 s^{2} k j, \quad 1 s 2 s^{2} 2 p_{1 / 2} k p_{3 / 2}$, $1 s 2 s^{2} 2 p_{3 / 2} k p_{1 / 2}$ for the total angular momenta $J$ $=1 / 2,3 / 2,5 / 2$ with $k$ running over all the eigenstates of Eq. (5) calculated on the generalized pseudospectral grid. The scattering wave function is obtained from Eq. (2) with $V$ $=V_{\text {res }}(N)-V_{\text {res }}(N-1)$. The initial state $\Psi_{0}=\Psi_{g}(N-1) \phi_{c}\left(\mathbf{r}_{N}\right)$ is the product of the ground state wave function $\left(1 s^{2} 2 s^{2}\right)$ of the $(N-1)$-electron system and the incident electron wave function $\phi_{c}(\mathbf{r})$, which is obtained by solving Eq. (5) numerically as a regular solution near the nucleus at a given incident energy $E_{e}$. In the asymptotic region, the two-component radial wave function of $\phi_{c}(\mathbf{r})$ is of the form,

$$
\lim _{r \rightarrow \infty}\left(\begin{array}{l}
G(r) \\
F(r)
\end{array}\right)=N\left(E_{e}\right)\left(\begin{array}{c}
\sin \alpha \\
\epsilon \cos \alpha
\end{array}\right),
$$

with 

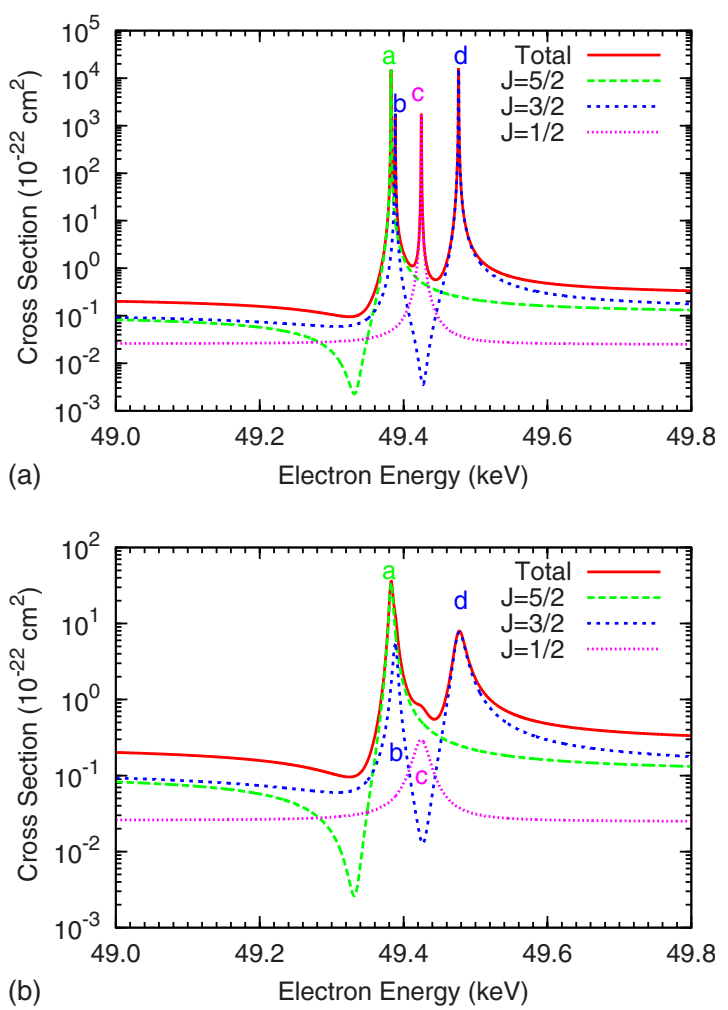

FIG. 1. (Color online) The total and partial radiative recombination cross sections of Be-like $\mathrm{Hg}$ ions without (upper panel) and with (lower panel) the radiative damping.

$$
\begin{gathered}
N\left(E_{e}\right)=\sqrt{\frac{2}{\pi} \frac{c^{3}}{\left(E_{e}+c^{2}\right) \sqrt{E_{e}^{2}+2 c^{2} E_{e}}},} \\
\epsilon=\sqrt{E_{e} /\left(E_{e}+2 c^{2}\right)}, \\
\alpha=k r+\eta \ln (k r)-\ell \pi / 2+\delta, \\
\eta=\frac{Z_{c}}{2 c}\left(\epsilon+\epsilon^{-1}\right) .
\end{gathered}
$$

Here, $\delta$ is the phase shift, $Z_{c}$ the effect charge and $k=\sqrt{2 E_{e}}$. The system decays to the ground state $\left|\Psi_{g}\right\rangle=\left|1 s^{2} 2 s^{2} 2 p_{1 / 2}\right\rangle$ or the first excited state $\left|\Psi_{e}\right\rangle=\left|1 s^{2} 2 s^{2} 2 p_{3 / 2}\right\rangle$ radiatively. Using Eq. (2), the reduced multipole transition matrix element for the radiative decay is expressed as

$$
\begin{aligned}
T_{c \rightarrow g, e}^{k}= & \left\langle\Psi_{g, e}\left\|T_{e, m}^{k}\right\| \Psi^{+}\right\rangle=\left\langle\Psi_{g, e}\left\|T_{e, m}^{k}\right\| \Psi_{0}\right\rangle \\
& +\sum_{j} \frac{\left\langle\Psi_{g, e}\left\|T_{e, m}^{k}\right\| \Psi_{j}\right\rangle\left\langle\Psi_{j}\|V\| \Psi_{0}\right\rangle}{E-E_{j}+i \eta}
\end{aligned}
$$

Here, $T_{e, m}^{k}$ is the electric and magnetic multipole transition operator [18]. The radiative transitions consist of two parts, the direct $\mathrm{RR}$ and the radiative decay through DR resonant states. The partial radiative recombination cross section is

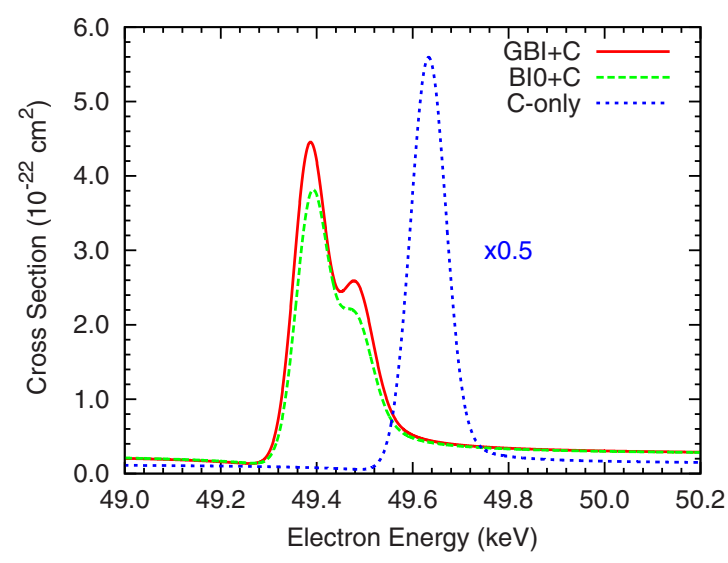

FIG. 2. (Color online) The convoluted RR cross sections with contributions of the electron-electron Coulomb interaction (dotted line), the Breit interaction in the low-frequency limit (dashed line) and the generalized Breit interaction (solid line).

$$
\sigma_{r r}^{k}=\frac{2 \pi^{2} \omega}{c^{3}(2 k+1)\left(1+E_{e} / 2 c^{2}\right) E_{e}}\left|T_{c \rightarrow g, e}^{k}\right|^{2},
$$

with $E_{e}$ the incident electron energy and $\omega$ the emitted photon energy. The total RR cross section is the sum of all the partial cross sections. The DR resonant states are

$$
\begin{aligned}
& \left|\Psi_{1 / 2}^{r}\right\rangle=\left|\left[1 s 2 s^{2} 2 p_{1 / 2}\right]_{1} 2 p_{3 / 2}\right\rangle_{J=1 / 2}, \\
& \left|\Psi_{0,3 / 2}^{r}\right\rangle=\left|\left[1 s 2 s^{2} 2 p_{1 / 2}\right]_{0} 2 p_{3 / 2}\right\rangle_{J=3 / 2}, \\
& \left|\Psi_{1,3 / 2}^{r}\right\rangle=\left|\left[1 s 2 s^{2} 2 p_{1 / 2}\right]_{1} 2 p_{3 / 2}\right\rangle_{J=3 / 2}, \\
& \left|\Psi_{5 / 2}^{r}\right\rangle=\left|\left[1 s 2 s^{2} 2 p_{1 / 2}\right]_{1} 2 p_{3 / 2}\right\rangle_{J=5 / 2} .
\end{aligned}
$$

The upper panel of Fig. 1 shows the total and partial RR cross sections near the resonant energies. The RR cross sections due to DR process are many orders of magnitude larger than that of the direct RR. If we convolute the spectra with the experimental energy resolution, we get an almost symmetric resonant profile which disagrees with the experimental observations [17]. Our theoretical treatment developed up to this point is not satisfactory yet and some further improvement is required. When we calculated the Green's function for all the bound-type configurations $\left\{\Phi_{b, i}\right\}$, we assumed that the life time comes only from the Auger processes. Since the radiative decay rates are much larger than the Auger decay rates for highly charged ions (HCI), we have to incorporate the radiative damping effect [19] in the calculations. We take into account the radiative damping in such a way that we add an imaginary energy $(-i \Gamma / 2)$ to the bound-state energy representing the radiative decay rate of a resonant state. The lower panel of Fig. 1 shows the improved results, in which the radiative decay rates are taken into account. Indeed the spectra show asymmetric distributions. The asymmetry arises from the typical bound-continuum interactions, which result in the Fano profiles.

After we convolute the cross sections with the experimental energy resolution of $77 \mathrm{eV}$ [20], we still observe the asymmetric line profile $(\mathrm{GBI}+\mathrm{C})$ in the resonant region as 
TABLE I. The resonant energies (in ev) and radiative decay rates (in ev) for B-like $\mathrm{Hg}$ ions. The configuration interaction coefficients of peaks $b$ and $d$ are also listed.

\begin{tabular}{lcccc}
\hline \hline Term & Quantity & Coulomb-only & $\mathrm{C}+\mathrm{BI} 0(\omega=0)$ & $\mathrm{C}+\mathrm{GBI}$ \\
\hline $\mathrm{a}$ & $E_{r}$ & -187733 & -187697 & -187702 \\
& $\Gamma$ & 5.4 & 5.4 & 5.4 \\
$\mathrm{c}$ & $E_{r}$ & -187720 & -187663 & -187659 \\
& $\Gamma$ & 24.0 & 23.9 & 23.9 \\
$\mathrm{~b}$ & $E_{r}$ & -187776 & -187694 & -187696 \\
& $\Gamma$ & 1.8 & 7.4 & 7.0 \\
& $\left(C_{0}, C_{1}\right)$ & $(0.561,-0.828)$ & $(0.884,-0.467)$ & $(0.876,-0.483)$ \\
& $\left(C_{+}, C_{-}\right)$ & $(0.189,-0.982)$ & $(0.297,-0.955)$ & $(0.277,-0.961)$ \\
$\mathrm{d}$ & $E_{r}$ & -187702 & -187611 & -187607 \\
& $\Gamma$ & 22.3 & 16.3 & $(0.483,0.876)$ \\
& $\left(C_{0}, C_{1}\right)$ & $(0.828,0.561)$ & $(0.467,0.884)$ & $(0.961,0.277)$ \\
& $\left(C_{+}, C_{-}\right)$ & $(0.982,0.189)$ & $(0.955,0.297)$ & 16.9 \\
\hline \hline
\end{tabular}

shown in Fig. 2. To investigate the Breit interaction contributions, we also plot the cross sections calculated with electron-electron Coulomb interaction only (C-only) or with $\mathrm{BIO}(\mathrm{BI} 0+\mathrm{C})$. Generally speaking, the Breit interaction shifts the resonant peaks to the lower energy side and increases the energy splitting as indicated in Table I. The Breit interaction also reduces the transition strengths by more than a factor of 2 as shown in Fig. 2, consistently with the recent experiment of Be-like $\mathrm{Bi}$ ions [21]. The GBI enhances the transition strengths by about $10 \%$ from the BI0 results. Our BI0 contributions to the transition energies are in good agreement with the calculations by Harman et al. [22].

As indicated in Table I, the radiative decay rates of peaks $a$ and $c$ are relatively insensitive to the Breit interaction, while the rates of peaks $b$ and $d$ depend evidently on the Breit interaction. The radiative decay is a single-electron process and thus generally insensitive to the Breit interaction. The decay rates of peaks $b$ and $d$ depend on the Breit interaction because the Breit interaction changes the linear combination of the two $J=3 / 2$ states.

In comparison with the partial cross sections of the different total angular momentum $J$, we assign the resonant peaks in Fig. 1 as peak $a$ originated from $J=5 / 2$, and peak $c$ originated from $J=1 / 2$. Peaks $b$ and $d$ are originated from the linear combinations of the two $J=3 / 2$ configurations as
$\left|\Psi_{d, b}\right\rangle=C_{0}\left|\Psi_{0,3 / 2}^{r}\right\rangle+C_{1}\left|\Psi_{1,3 / 2}^{r}\right\rangle$. Conventionally, we assign a peak as a state with the largest coefficient. Following this rule, we should assign peak $d$ as $\left|\Psi_{0,3 / 2}^{r}\right\rangle$ for Coulomb only and reassign it as $\left|\Psi_{1,3 / 2}^{r}\right\rangle$ when GBI is taken into account as indicated in Table I. The radiative decay rates of state $d$ are always larger than that of state $b$ regardless of whether the GBI is included not. This implies that there may be another way to classify the two peaks better. If we define $\left|\Psi_{ \pm}\right\rangle$ $=\left(\left|\Psi_{0,3 / 2}^{r}\right\rangle \pm\left|\Psi_{1,3 / 2}^{r}\right\rangle\right) / \sqrt{2}$, and rewrite $\left|\Psi_{d, b}\right\rangle=C_{+}\left|\Psi_{+}^{-}\right\rangle$ $+C_{-}\left|\Psi_{-}\right\rangle$, we assign peak $d$ as state $\left|\Psi_{+}\right\rangle$and peak $b$ as state $\left|\Psi_{-}\right\rangle$for all the three cases as shown in Table I. Although it is convenient to use $\left|\Psi_{0,3 / 2}^{r}\right\rangle$ and $\left|\Psi_{1,3 / 2}^{r}\right\rangle$ as configurations for the calculation, $\left|\Psi_{ \pm}\right\rangle$give better representation for the classification of the strongly mixed states. This situation is similar to the case of classification of the doubly excited states of $|2 s n p\rangle$ and $|2 p n s\rangle$ of helium atoms [23].

Once we obtain the radiative recombination cross sections as shown in the lower panel of Fig. 1, we fit the spectra to the following Fano line profile [24] as

$$
F(E)=\frac{A}{q^{2} \Gamma}\left[\frac{(\epsilon+q)^{2}}{\epsilon^{2}+1}-1\right],
$$

with $\epsilon=2\left(E-E_{r}\right) / \Gamma$. Here, $E_{r}$ is the resonant energy, $\Gamma$ the width of the resonant state, and $A$ the relative transition

TABLE II. The Fano $q$ parameter and the relative strength $A$ (normalized to the strong line) for all the possible transitions. The measured and MCDF calculated $q$ parameters [17] are also listed for comparison.

\begin{tabular}{lccccc}
\hline \hline Trans. & Term & $\begin{array}{c}\mathrm{a} \\
(\mathrm{J}=5 / 2)\end{array}$ & $\begin{array}{c}\mathrm{b} \\
(\mathrm{J}=3 / 2)\end{array}$ & $\begin{array}{c}\mathrm{c} \\
(\mathrm{J}=1 / 2)\end{array}$ & $\begin{array}{c}\mathrm{d} \\
(3 / 2)\end{array}$ \\
\hline$\rightarrow\left|\Psi_{g}\right\rangle$ & $q$ & $>100$ & 19.4 & -5.9 & 6.9 \\
& $(A)$ & $(0.01)$ & $(0.08)$ & $(0.03)$ & $(0.61)$ \\
$\rightarrow\left|\Psi_{e}\right\rangle$ & $q$ & 20.1 & -11.9 & 2.3 & $>100$ \\
& $(A)$ & $(1.00)$ & $(0.11)$ & $(0.01)$ & $(0.05)$ \\
Ours & $q$ & 20.1 & & & 6.9 \\
Measured [17] & $q$ & $18.2(6.6)$ & & & $6.7(0.6)$ \\
MCDF [17] & $q$ & 13 & & & 7.3 \\
\hline \hline
\end{tabular}


strength of the resonant state and $q$ the Fano parameter. The fitted $E_{r}$ and $\Gamma$ are so close to the values listed in Table I that we list only $A$ and $q$ in Table II. For comparison, we also list the measured $q$ parameters and the calculated ones [17] based on the multiconfiguration-Dirac-Fock (MCDF) method, in which the coupling between the resonant state and the continuum was treated to the lowest order. From the relative transition strengths, we see that the dominant contributions come from the transitions from state $a$ to the first excited state $(\mathrm{A}=1.00)$, from state $d$ to the ground state (A $=0.61)$, and from state $b$ to the first excited state $(A=0.11)$. The contribution of the others is less than $10 \%$. Thus, from our calculations, we identify the two observed peaks as the transition from state $a$ to the first excited state and the transition from state $d$ to the ground state. Our calculated $q$ parameters are also in reasonable agreement with the measurements. The present $q$ parameters agree with the experiment ones better than the MCDF calculations because we have taken into account all the bound-bound and bound- continuum interactions. The difference between ours and MCDF calculations can be attributed to the high order contributions missing in the MCDF calculations.

To summarize, we developed a method to calculate the Green's function of multielectron ions with one electron in continuum. As an example, we studied the asymmetric line profiles observed in the radiative recombination in HCIs and found that the radiative damping plays an important role. The present method can also be used to study other dynamic processes of multielectron systems.

\section{ACKNOWLEDGMENTS}

This work is supported by Grant-in-Aids for Scientific Research (C) from the Japan Society for the Promotion of Science. The numerical calculations have been carried out by T2K-Tsukuba System in Center for Computational Sciences, University of Tsukuba.
[1] X. M. Tong, J. M. Li, L. Kissel, and R. H. Pratt, Phys. Rev. A 42, 1442 (1990).

[2] X. M. Tong and Shih-I Chu, Phys. Rev. A 55, 3406 (1997).

[3] P. G. Burke and K. T. Taylor, J. Phys. B 8, 2620 (1975).

[4] T. W. Gorczyca, N. R. Badnell, and D. W. Savin, Phys. Rev. A 65, 062707 (2002).

[5] X. M. Tong, K. Hino, and N. Toshima, Phys. Rev. Lett. 97, 243202 (2006).

[6] X. M. Tong and Shih-I Chu, Phys. Rev. A 61, 031401(R) (2000).

[7] X. M. Tong, T. Shirahama, K. Hino, and N. Toshima, Phys. Rev. A 75, 052711 (2007).

[8] X. M. Tong and Shih-I Chu, Phys. Rev. A 57, 452 (1998).

[9] I. P. Grant and B. J. McKenzie, J. Phys. B 13, 2671 (1980).

[10] J. Wang, Shih-I Chu, and C. Laughlin, Phys. Rev. A 50, 3208 (1994).

[11] X. M. Tong and S. I. Chu, Chem. Phys. 217, 119 (1997).

[12] J. Perie and G. Jolicard, J. Phys. B 26, 4491 (1993).

[13] G. Gaigalas and S. Fritzsche, Comput. Phys. Commun. 148, 349 (2002).

[14] B. Simon, Phys. Lett. A 71, 211 (1979).

[15] J. M. Rost, K. Schulz, M. Domke, and G. Kaindl, J. Phys. B 30, 4663 (1997).
[16] C. W. McCurdy, D. A. Horner, T. N. Rescigno, and F. Martin, Phys. Rev. A 69, 032707 (2004).

[17] A. J. Gonzalez Martinez, J. R. Crespo Lopez-Urrutia, J. Braun, G. Brenner, H. Bruhns, A. Lapierre, V. Mironov, R. Soria Orts, H. Tawara, M. Trinczek, J. Ullrich, and J. H. Scofield, Phys. Rev. Lett. 94, 203201 (2005).

[18] I. P. Grant, J. Phys. B 7, 1458 (1974).

[19] P. C. W. Davies and M. J. Seaton, J. Phys. B 2, 757 (1969).

[20] H. Tobiyama, H. Nohara, A. P. Kavanagh, N. Nakamura, H. Watanabe, H. A. Sakaue, Y. Li, D. Kato, F. J. Curell, C. Yamada, and S. Ohtani, J. Phys.: Conf. Ser. 58, 239 (2007).

[21] N. Nakamura, A. P. Kavanagh, H. Watanabe, H. A. Sakaue, Y. Li, D. Kato, F. J. Currell, and S. Ohtani, Phys. Rev. Lett. 100, 073203 (2008)

[22] Z. Harman, I. I. Tupitsyn, A. N. Artemyev, U. D. Jentschura, C. H. Keitel, J. R. Crespo Lopez-Urrutia, A. J. Gonzalez Martinez, H. Tawara, and J. Ullrich, Phys. Rev. A 73, 052711 (2006).

[23] J. W. Cooper, U. Fano, and F. Prats, Phys. Rev. Lett. 10, 518 (1963).

[24] S. Schippers, S. Kieslich, A. Muller, G. Gwinner, M. Schnell, A. Wolf, A. Covington, M. E. Bannister, and L. B. Zhao, Phys. Rev. A 65, 042723 (2002). 